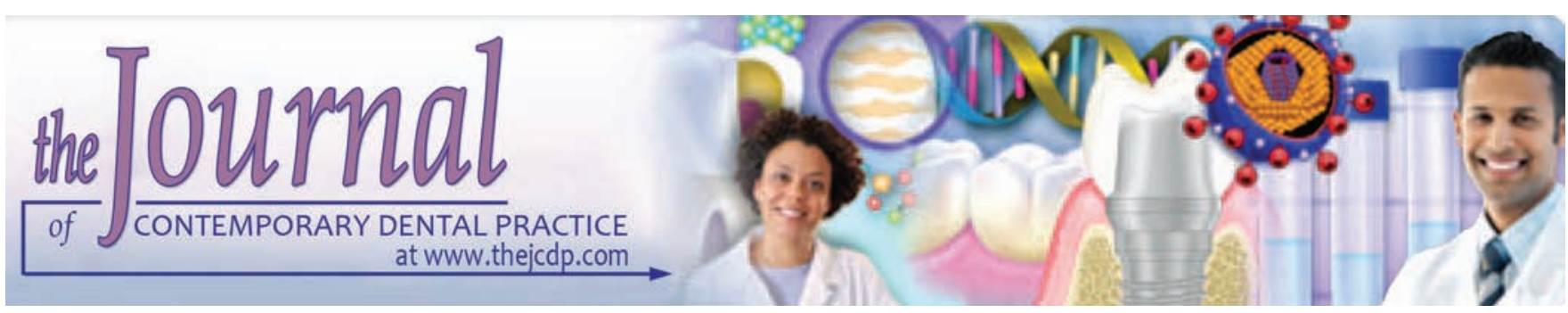

\title{
Assessment of Proliferative Potential of Odontogenic Keratocyst and Dentigerous Cyst using Podoplanin: An Immunohistochemical Study
}

${ }^{1}$ Sandeep Gupta, ${ }^{2}$ Aparna Paliwal, ${ }^{3}$ Nidhi Choudaha, ${ }^{4}$ Anish Gupta, ${ }^{5}$ Prashant Rao, ${ }^{6}$ Shekhar Grover

\begin{abstract}
Introduction: Odontogenic cysts are commonly encountered lesions among head and neck pathologies. Odontogenic keratocyst $(\mathrm{OKC})$ has unique features of recurrence and local aggressiveness. Podoplanin (PDP) is a lymphatic endothelial marker and is shown to be expressed in a variety of tissues. Hence, we planned to assess the significance of PDP in OKC and dentigerous cyst (DC).
\end{abstract}

Materials and methods: The present study included assessment of immunoexpression of PDP in OKC and DC. Twenty specimens each of OKC and DC were included in the present study and were stained with D2-40 antibody. All the sections were analyzed and were categorized as negative staining, weakly positive staining, and strongly positive staining. All the results were analyzed by Statistical Package for the Social Sciences (SPSS) software.

Results: We detected PDP-positive staining in the cell membrane and cytoplasm of the cells of basal cell layer and suprabasal cell layers. In DC cases, we observed positive staining only in cases associated with inflammation.

Conclusion: Podoplanin does play a significant role in enhancing the local invasive and neoplastic properties of OKC.

\footnotetext{
${ }^{1,3}$ Department of Oral Pathology and Microbiology, Bhabha College of Dental Sciences, Bhopal, Madhya Pradesh, India

${ }^{2}$ Department of Oral Pathology and Microbiology, RKDF Dental College and Research Centre, Bhopal, Madhya Pradesh, India

${ }^{4}$ Department of Oral Pathology and Microbiology, People's Dental Academy, Bhopal, Madhya Pradesh, India

${ }^{5}$ Department of Oral Pathology and Microbiology, Bharti Vidyapeeth Deemed University, Dental College and Hospital Pune, Maharashtra, India

${ }^{6}$ Department of Public Health Dentistry, Maharaja Agrasen Institute of Management Studies, New Delhi, India
}

Corresponding Author: Sandeep Gupta, Department of Oral Pathology and Microbiology, Bhabha College of Dental Sciences Bhopal, Madhya Pradesh, India, Phone: +919501544877 e-mail: doc.sandeepgupta@gmail.com
Clinical significance: Podoplanin expression in OKC is potentially associated with moderate invasive nature of the neighboring structures.

Keywords: Dentigerous cyst, Odontogenic keratocyst, Podoplanin.

How to cite this article: Gupta S, Paliwal A, Choudaha N, Gupta A, Rao P, Grover S. Assessment of Proliferative Potential of Odontogenic Keratocyst and Dentigerous Cyst using Podoplanin: An Immunohistochemical Study. J Contemp Dent Pract 2017;18(12):1173-1176.

Source of support: Nil

Conflict of interest: None

\section{INTRODUCTION}

One of the commonly encountered lesions of the head and neck region is odontogenic cysts (OOCs). They comprise a significant component of biopsies received by any of the pathologists and pathology laboratories. ${ }^{1}$ A wide range of morphologic variability is exhibited by these groups of lesions ranging from the small innocuous lesion to highly aggressive and potentially destructive large lesions that have capability and potential of getting converted into malignancy. One such odontogenic lesion is OKC. ${ }^{2-4}$ Odontogenic keratocyst is one of the commonly occurring OOCs that have certain unique histopathologic and clinical features that attract many keen researchers and pathologists. Odontogenic keratocyst is a developmental cyst that rises from remnants of dental lamina and was first classified by Philipsen in 1956. Due to formation of compartments within it, it has the potential of showing high growth rate and high recurrence rate., ${ }^{1,5}$

Podoplanin is a lymphatic endothelial marker and is shown to be expressed in a variety of tissues (both in physiologic and pathologic settings) by various researchers. ${ }^{6,7}$ Marked expression of PDP is shown to be 
positive in a wide variety of OOCs and tumors including OKC. ${ }^{8}$ Various researchers have hypothesized that PDP plays a significant role in the tumor proliferation and invasion process. ${ }^{8}$ Hence, we planned to assess the significance of PDP in OKC and DC.

\section{MATERIALS AND METHODS}

The present study was conducted in the Department of Oral Pathology of the Bhabha College of Dental Sciences Bhopal, Madhya Pradesh, India and included assessment of paraffin-embedded cases of OKC and DC. A total of 40 cases reported from the Department of Oral Pathology were included in the present study, out of which 20 were of OKC and 20 were of DC. All the specimens were obtained from the archives of the Department of Oral Pathology. Only parakeratinized OKCs were included in the present study. All the cases were diagnosed based on their clinical and histopathological findings. Immunohistochemical staining of all the sections was done using mouse monoclonal D2-40 antibody which stains for PDP marker. Ethical approval was taken from the institutional ethical committee, and written consent was obtained after explaining in detail the entire research protocol. Complete demographic details of all the cases were recorded separately.

\section{Immunohistochemical Protocol}

Sections measuring $3 \mu \mathrm{m}$ of all the cases were cut under a microtome followed by obtaining them on glass slide from wax water bath and dewaxing. All the dewaxed sections were immersed in absolute alcohol which had $0.3 \%$ hydrogen peroxide solution. The sections were immersed for 15 to 20 minutes for blocking the endogenous peroxide activity. The sections were washed with normal saline followed by immersion into citrate buffer solution. After removal, the sections were subjected to heat in a microwave oven for retrieving the antigen. After antigen retrieval, mouse monoclonal antihuman D2-40 (monoclonal anti-PDP) was added to the sections for 50 minutes followed by prediluted antimouse immunoglobulin $\mathrm{G}$ antibody conjugated with peroxidase for 50 minutes. After this, the sections were immersed in $0.05 \%$ 3, 3'-diaminobenzidine tetrahydrochloride followed by application of Tris- $\mathrm{HCl}$ buffer. After the completion of immunostaining procedure, the sections were counterstained with Mayer's hematoxylin. For assessment of reactivity of PDP, following criteria were used as described previously in the literature?

- $(-)$ Negative

- (+) Weakly positive

- (++) Strongly positive.

All the results were analyzed by SPSS software version 16.0. Chi-square test and Student's t-test were used for
Table 1: Mean values and SD of the intensity of the PDP immunostaining $(A)$, percentage of PDP-positive odontogenic cells $(B)$, and the final immunostaining score $(A+B)$ observed in OKCs and DCs

\begin{tabular}{lcll}
\hline & $\begin{array}{l}\text { Immunostaining } \\
\text { intensity }(A)\end{array}$ & $\begin{array}{l}\text { Percentage of } \\
\text { PDP-positive } \\
\text { cells }(B)\end{array}$ & $\begin{array}{l}\text { Final score } \\
(A+B)\end{array}$ \\
Group & Mean $\pm S D$ & Mean $\pm S D$ & Mean \pm SD \\
\hline OKC $(\mathrm{n}=20)$ & $3.20 \pm 0.75$ & $2.60 \pm 0.49$ & $5.80 \pm 0.98$ \\
DCs $(\mathrm{n}=20)$ & $1.20 \pm 0.75$ & $1.60 \pm 0.49$ & $2.80 \pm 1.17$ \\
t-value & 4.869 & 3.726 & 5.256 \\
p-value & $<0.05$ & $<0.05$ & $<0.05$ \\
\hline
\end{tabular}

SD: Standard deviation

Table 2: Immunostaining for PDP in OKC and DC

\begin{tabular}{lllll}
\hline Cyst & Number & Negative & Weakly positive & Strongly positive \\
\hline OKC & 20 & 2 & 3 & 15 \\
DC & 20 & 3 & $3^{*}$ & $14^{*}$ \\
\hline
\end{tabular}

*Associated with inflammation in the connective tissue stroma

the assessment of the level of significance; $\mathrm{p}<0.05$ was considered as statistically significant.

\section{RESULTS}

Tables 1 and 2 show the immunostaining of PDP in OKC and DC (Fig. 1). In most of the cells of the basal and suprabasal layer, the expression of PDP in OKCs was strongly positive. Furthermore, positive immunostaining was observed in the epithelial cell nests and basal cell proliferations. In the peripheral cells of the daughter cyst, slight immunoreactivity was found to be positive for PDP. Two cases of OKC and two cases of DC were found to be negative for the staining of PDP. In PDP-positive cases among DC group, the positive staining was found to be associated with inflammation in the connective tissue stroma (Graph 1).

\section{DISCUSSION}

The PDP is a lymphatic endothelium marker and is broadly utilized as a specific marker for lymphangiogenesis. Notwithstanding, articulation of PDP is upmanaged in various types of cancerous lesions. Odontogenic keratocyst is one of the most common types of lesion of odontogenic origin. Histopathologically, it is characterized by stratified squamous epithelial lining comprising six to ten layers and covered by parakeratinized corrugated epithelium. ${ }^{10,11}$

Other histopathologic findings in the epithelial lining of OKC include basal layer palisading with the absence of rete ridges. ${ }^{12}$

In the present study, we observed high expression of PDP in OKC and association of inflammation with the PDP reactivity in the DC cases (Table 1). This might reflect the invasive nature of PDP as demonstrated 


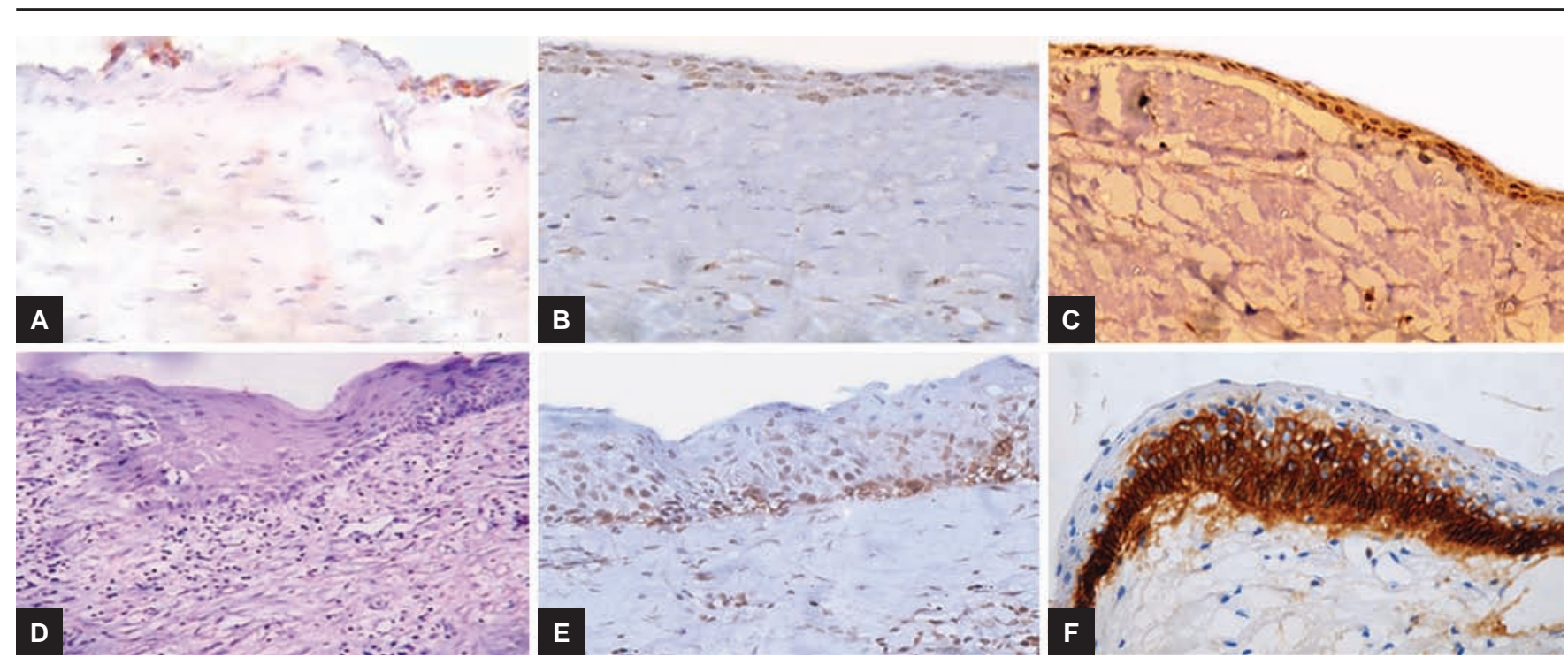

Figs 1A to F: Immunostaining for PDP showing: (A) Negative staining; (B) weakly positive; (C) strongly positive in DC; (D) negative staining; (E) weakly positive; and (F) strongly positive in OKC

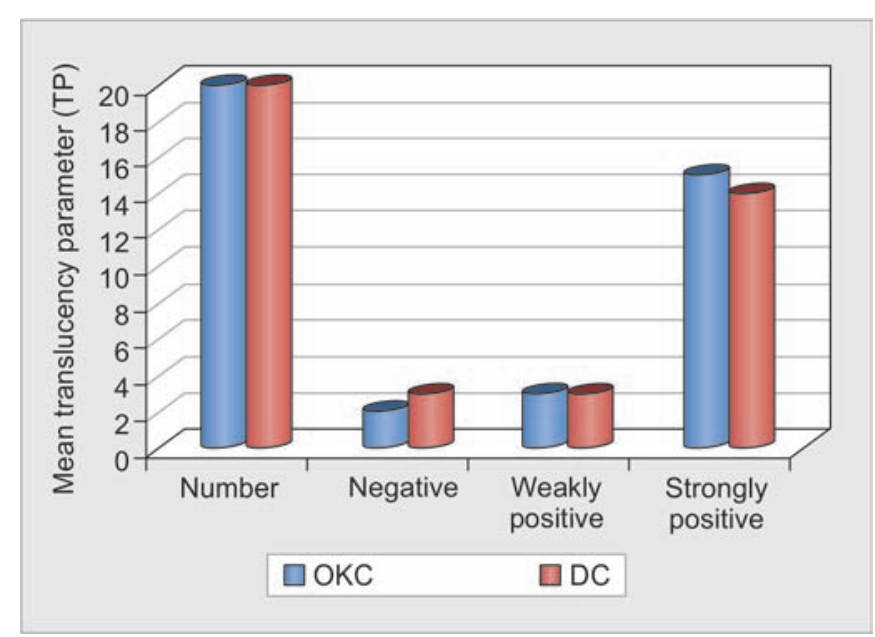

Graph 1: Immunostaining for PDP in OKC and DC

by high PDP activity. Our results were in correlation with the results obtained by Okamoto et $\mathrm{al}^{9}$ who also observed similar findings in their study. Zustin et $\mathrm{al}^{13}$ evaluated the expression of PDP in human tooth germ tissue and odontogenic pathologies. They analyzed nine human tooth germ specimens along with 70 ideal, noncarious permanent teeth extracted because of orthodontic reasons. They assessed D-reactivity. Among odontogenic pathologies, they assessed radicular cyst specimens, follicular cyst specimens along with OKC ameloblastomas, and adenomatoid odontogenic tumor specimens. In majority of the epithelial and ectomesenchymal cells, they observed positive PDP expression in specimens of human germ tooth tissues. Furthermore, positive PDP expression was observed in the invasive front of the odontogenic lesions. From the results, they concluded that PDP might be an important component involved in the pathologic lesions involving the odontogenic apparatus.
Okamoto et $\mathrm{al}^{9}$ decided the value of PDP for renaming of the OKC from cyst to tumor status. Paraffin-installed tissue examples of $57 \mathrm{OKCs}$ [46 keratocystic odontogenic tumors (KCOTs) and 11 orthokeratinized OOCs] and 15 DCs were inspected immunohistochemically by utilizing antibody directed against PDP. Majority of the basal and suprabasal layers exhibited immunohistochemical reactivity for PDP in their cytoplasm and cell membrane. They also observed positive immunostaining in the basal cell proliferative areas along with epithelial cell nests and daughter cysts (peripheral cells only) in the connective tissue capsule of KCOTs. The authors observed strong expression of PDP in KCOTs in comparison with OOCs and emphasized that PDP has a definitive role in tumor invasive behavior.

Friedrich et $\mathrm{al}^{14}$ studied the PDP expression in KCOTs related with nevoid basal cell carcinoma syndrome (NBCCS). Chronicled paraffin-implanted tissues from six KCOTs from patients with known NBCCS were broke down immunohistochemically with antibodies to PDP (D2-40) and P63. They observed a constant direct immunoreactivity of basal epithelial cells for PDP in all cases. The recoloring power was solid and did not contrast from that for KCOT in already revealed sporadic cases. Solid atomic p63 expression was distinguished in basal cell layers and lessened in suprabasal layers. The KCOTs displayed upgraded PDP expression in a clinical setting of NBCCS. In spite of the fact that the natural elements of PDP have not yet been completely perceived, the overexpression of this protein is equipped for advancing the arrangement of lengthened cell expansions and expanding grip and relocation of provocative cells. Podoplanin expression in KCOT is conceivably connected with moderate attack of the nearby structures and the 
outstanding successive neighborhood tumor repeats of this odontogenic tumor.

\section{CONCLUSION}

The PDP does play a significant role in enhancing the local invasive and neoplastic properties of OKC. However, as there is a paucity of literature in relation of PDN in OOCs, we recommend future studies.

\section{REFERENCES}

1. Philipsen HP, Reichart PA. Classification of odontogenic tumours. A historical review. J Oral Pathol Med 2006 Oct;35(9):525-529.

2. Hauer A. Ein Cholesteatomimlinken Unterkieferuntereinemretinierten weisheitszahn. Zeitsschrift fur Stomatologie 1926;24:40-59.

3. Barnes L, Eveson JW, Reichart P, Sidransky D, editors. Pathology and Genetics of Head and Neck Tumours. Lyon: IARC Press; 2005.

4. Toller P. Origin and growth of cysts of the jaws. Ann R Coll Surg Engl 1967 May;40(5):306-336.

5. Robinson HB. Primordial cysts versus keratocysts. Oral Surg Oral Pathol Oral Med 1975 Sep;40(3):362-364.

6. Astarita JL, Acton SE, Turley SJ. Podoplanin: emerging functions in development, the immune system, and cancer. Front Immunol 2012 Sep;3:283.

7. Tsuneki M, Maruyama S, Yamazaki M, Cheng J, Saku T. Podoplanin expression profiles characteristic of odontogenic tumour-specific tissue architectures. Pathol Res Pract 2012 Mar;208(3):140-146.
8. Wicki A, Lehembre F, Wick N, Hantusch B, Kerjaschki D, Christofori G. Tumour invasion in the absence of epithelialmesenchymal transition: podoplanin-mediated remodeling of the actin cytoskeleton. Cancer Cell 2006 Apr;9(4): 261-272.

9. Okamoto E, Kikuchi K, Miyazaki Y, González-Alva P, Oku Y, Tanaka A, Yoshida N, Fujinami M, Ide F, Sakashita H, et al. Significance of podoplanin expression in keratocystic odontogenic tumor. J Oral Pathol Med 2010 Jan;39(1):110-114.

10. Kato Y, Kaneko M, Sata M, , Fujita N, Tsuruo T, Osawa M. Enhanced expression of aggress (T1\&/podoplanin), a plateletaggregation-inducing factor in lung squamous cell carcinoma. Tumor Biol 2005 Jul-Aug;26(4):195-200.

11. Martin-Villar E, Scholl FG, Gamallo C, Yurrita MM, Guerra MM, Cruces J, Quintanilla M. Characterization of human PA2.26 antigen (T1alpha-2, podoplanin), a small membrane mucin induced in oral squamous cell carcinomas. Int J Cancer 2005 Mar;113(6):899-910.

12. Schacht $\mathrm{V}$, RamirezMI,Hong $Y K$, HirakawaS, Feng D,Harvey N, Williams M, Dvorak AM, Dvorak HF, Oliver G, et al. T1alpha podoplanin deficiency disrupts normal lymphatic vasculature formation and causes lymphedema. EMBO J 2003 Jul;22(14):3546-3556.

13. Zustin J, Scheuer HA, Friedrich RE. Podoplanin expression in human tooth germ tissues and cystic odontogenic lesions: an immunohistochemical study. J Oral Pathol Med 2010 Jan;39(1):115-120.

14. Friedrich RE, Scheuer HA, Zustin J. Expression of podoplanin in nevoid basal cell carcinoma syndrome-associated keratocystic odontogenic tumours. Anticancer Res 2012 May;32(5):2125-2127. 